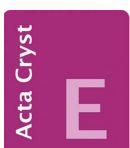

CRYSTALLOGRAPHIC COMMUNICATIONS

ISSN 2056-9890

Received 18 February 2019

Accepted 21 February 2019

Edited by A. Van der Lee, Université de Montpellier II, France

Keywords: crystal structure; crystal growth; $\beta$ xenophyllite structure; $\mathrm{K}_{3} \mathrm{Ni}_{6} \mathrm{Fe}\left(\mathrm{PO}_{4}\right)_{6} ; \mathrm{X}$-ray diffraction; othophosphate; solid-state reaction synthesis.

CCDC reference: 1898755

Supporting information: this article has supporting information at journals.iucr.org/e

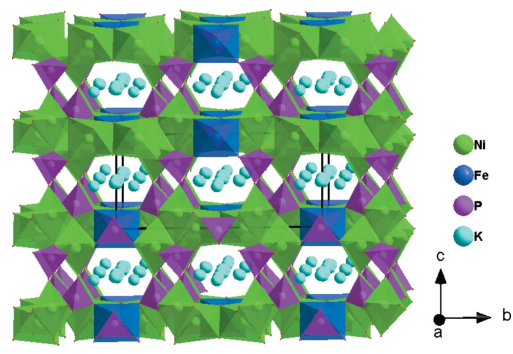

OPEN $\odot$ ACCESS

\section{Crystal structure of a new tripotassium hexanickel iron hexaphosphate}

\author{
Said Ouaatta,* Abderrazzak Assani, Mohamed Saadi and Lahcen El Ammari \\ Laboratoire de Chimie Appliquée des Matríiaux, Centre des Sciences des Matériaux, Faculty of Sciences, Mohammed V \\ University in Rabat, Avenue Ibn Batouta, BP 1014, Rabat, Morocco. *Correspondence e-mail: saidouaatta87@gmail.com
}

A new potassium-nickel iron phosphate, $\mathrm{K}_{3} \mathrm{Ni}_{6} \mathrm{Fe}\left(\mathrm{PO}_{4}\right)_{6}$, has been synthesized by solid-state reaction and structurally characterized by single-crystal X-ray diffraction and qualitative energy dispersive X-ray spectroscopy (EDS) analysis. The structure is built up by $\left[\mathrm{FeO}_{6}\right],\left[\mathrm{PO}_{4}\right]$, and $\left[\mathrm{NiO}_{6}\right]$ coordination polyhedra, which are linked to each other by edge and corner sharing to form zigzag layers parallel to the $a b$ plane. These layers are interconnected by $\left[\mathrm{PO}_{4}\right]$ tetrahedra and $\left[\mathrm{NiO}_{6}\right]$ octahedra via common corners, leading to a three-dimensional framework delimiting large channels running along the [100] direction in which the $\mathrm{K}^{+}$ cations are localized.

\section{Chemical context}

Iron-based phosphates are widely studied materials today. They present a promising field for various applications such as electronics (Saw et al., 2014), ferroelectrics (Lazoryak et al., 2004), magnetic materials (Hatert et al., 2004; Essehli et al., 2015) and catalytic processes (Moffat, 1978). The introduction of alkali metals into these phosphates materials can be of great interest to improve the ion-conduction properties for applications in rechargeable alkaline batteries (La Parola et al., 2018; Orikasa et al., 2016). The present work is part of our activity devoted particularly to the investigation of new materials based on phosphates belonging to the $A_{2} \mathrm{O}-M \mathrm{O}-$ $\mathrm{Fe}_{2} \mathrm{O}_{3}-\mathrm{P}_{2} \mathrm{O}_{5}(A=$ an alkali metal; $M=$ divalent cation $)$ quaternary system, which could have interesting ionic conductivity or magnetic proprieties. We report herein on the synthesis and structural characterization by single crystal $\mathrm{X}$-ray diffraction of a new potassium nickel iron phosphate with formula $\mathrm{K}_{3} \mathrm{Ni}_{6} \mathrm{Fe}\left(\mathrm{PO}_{4}\right)_{6}$.

\section{Structural commentary}

The asymmetric unit of the title compound, $\mathrm{K}_{3} \mathrm{Ni}_{6} \mathrm{Fe}\left(\mathrm{PO}_{4}\right)_{6}$, consists of two $\left[\mathrm{NiO}_{6}\right]$ octahedra, one $\left[\mathrm{FeO}_{6}\right]$ octahedron, two $\left[\mathrm{PO}_{4}\right]$ tetrahedra, and three $\mathrm{K}$ atoms, as shown in Fig. 1. One $\mathrm{Ni}^{2+}, \mathrm{Fe}^{3+}, \mathrm{P}^{5+}$, two $\mathrm{K}^{+}$cations and two of the seven oxygen atoms lie on special positions. The Ni2 atom occupies Wyckoff position $4 g(2)$, the $\mathrm{Fe}$ atom is localized on the $2 a(2 / m)$ Wyckoff position, $\mathrm{P} 2, \mathrm{~K} 1, \mathrm{~K} 3, \mathrm{O} 6$ and $\mathrm{O} 7$ are positioned on $4 i$ $(m)$ sites. The octahedral coordination sphere of the nickel(II) cation more distorted than that of the iron(III) atom, with average $<\mathrm{Ni}-\mathrm{O}>$ distances of 2.066 and $2.119 \AA$ for $\mathrm{Ni} 1$ and $\mathrm{Ni} 2$, respectively. The mean $\left\langle\mathrm{P}-\mathrm{O}>\right.$ distance in the two $\mathrm{PO}_{4}$ tetrahedra is equal to $1.547 \AA$ for $\mathrm{P} 1$ and $1.543 \AA$ for $\mathrm{P} 2$. 


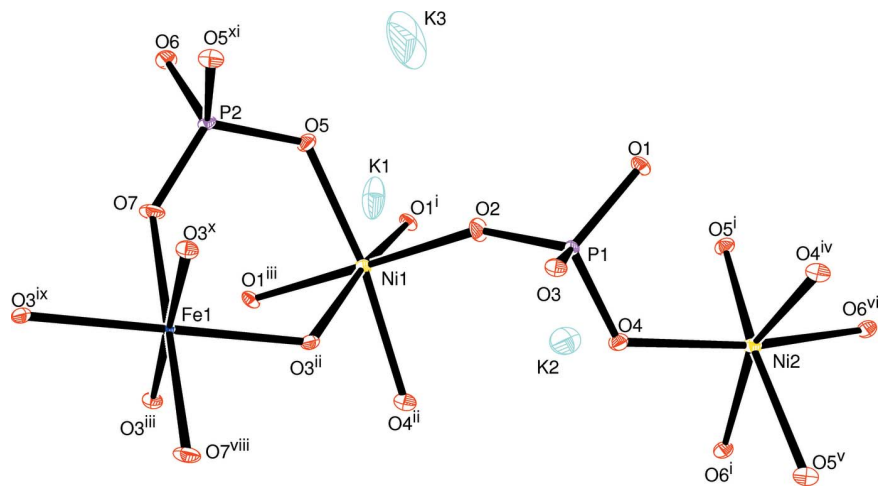

Figure 1

Molecular structure of the title compound with the atom-labelling scheme. Displacement ellipsoids are drawn at the $50 \%$ probability level. Symmetry codes: (i) $-x+\frac{1}{2},-y+\frac{3}{2},-z+1$; (ii) $-x+1, y,-z+1$; (iii) $x, y$, $z-1$; (iv) $-x+1, y,-z+2$; (v) $x+\frac{1}{2},-y+\frac{3}{2}, z+1$; (vi) $x+\frac{1}{2}, y+\frac{1}{2}, z+1$; (vii) $-x+1,-y+1,-z$; (viii) $x,-y+1, z-1$; (ix) $-x+1,-y+1,-z+1$; (x) $x,-y+1, z$; (xi) $x, y, z+1$; (xii) $-x+\frac{1}{2},-y+\frac{3}{2},-z$; (xiii) $-x,-y+1$, $-z$; (xiv) $x,-y+2, z$.

The Fe atoms are coordinated octahedrally with an average $<\mathrm{Fe}-\mathrm{O}>$ distance of $2.038 \AA$. The structure of the title compound is built up from two types of nickel sites and one iron site, each with an octahedral coordination environment, $\left[\mathrm{Ni1O}_{6}\right],\left[\mathrm{Ni}_{2} \mathrm{O}_{6}\right]$ and $\left[\mathrm{FeO}_{6}\right]$, besides two independent phosphor tetrahedra $\left[\mathrm{P}^{2} \mathrm{O}_{4}\right]$ and $\left[\mathrm{P}_{2} \mathrm{O}_{4}\right]$. Edge-sharing $\left[\mathrm{Ni} 2 \mathrm{O}_{6}\right]$ octahedra build up a dimeric $\left[\mathrm{Ni}_{2} \mathrm{O}_{10}\right]$ unit. Two $\left[\mathrm{P} 2 \mathrm{O}_{6}\right]$ octahedra are connected to the $\left[\mathrm{Ni}_{2} \mathrm{O}_{10}\right]$ dimer by sharing edges to form an $\left[\mathrm{Ni}(2)_{2} \mathrm{P}(2)_{2} \mathrm{O}_{12}\right]$ unit, which alternates with an $\left[\mathrm{FeO}_{6}\right]$ octahedron to establish an infinite chain along the [100] direction (Fig. 2). In addition, the association between the $\left[\mathrm{P} \mathrm{O}_{4}\right]$ tetrahedra and the $\left[\mathrm{Ni1O}_{6}\right]$ octahedra by means of edge-sharing allows the formation of a zigzag chain running parallel to the [100] direction. Each of the $\mathrm{P} \mathrm{O}_{4}$ tetrahedra

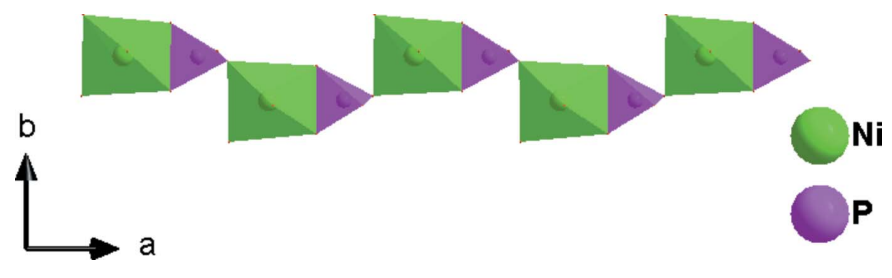

Figure 3

Corner- and edge-sharing $\left[\mathrm{P} \mathrm{O}_{4}\right]$ tetrahedra and $\left[\mathrm{Ni}^{1} \mathrm{O}_{6}\right]$ octahedra forming a zigzag shape chain running parallel to [100]

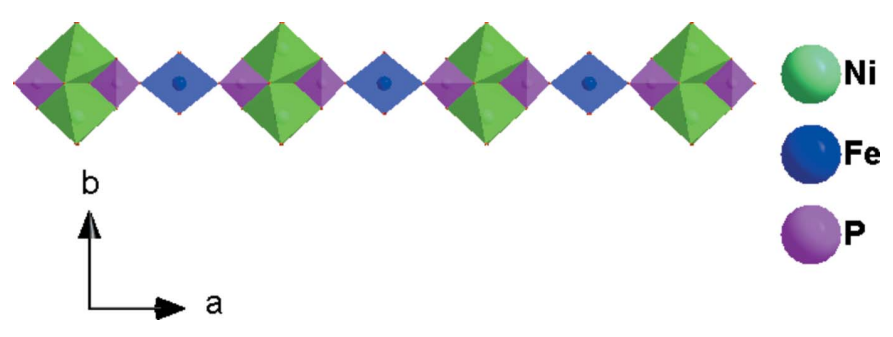

Figure 2

A chain formed by sharing edges and corners of $\left[\mathrm{Ni}_{2} \mathrm{O}_{10}\right]$ dimers, $\left[\mathrm{P}_{2} \mathrm{O}_{4}\right]$ tetrahedra and $\left[\mathrm{FeO}_{6}\right]$ octahedra along the [100] direction

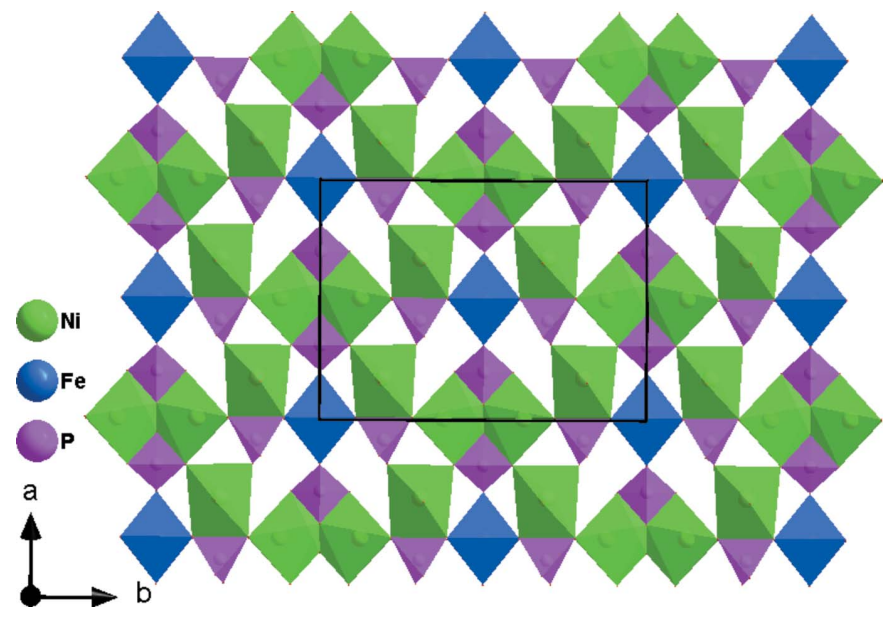

Figure 4

View along the $c$ axis of corner- and edge-sharing $\left[\mathrm{PO}_{4}\right]$ tetrahedra and $\left[\mathrm{NiO}_{6}\right]$ octahedra forming a layer parallel to the $a b$ plane.

and $\mathrm{Ni}_{6}$ octahedra, both belonging to the same layer, share vertices with $\mathrm{Ni1O}_{6}$ and $\mathrm{P}_{1} \mathrm{O}_{4}$, respectively, of the adjacent one (Fig. 3). The two types of chain linkages lead to the formation of layers parallel to the $a b$ plane (Fig. 4). One vertex of an $\mathrm{NilO}_{6}$ octahedron belonging to one layer is shared with a $\mathrm{P}_{1} \mathrm{O}_{4}$ vertex of the neighbouring layer. This configuration leads to a three-dimensional centrosymmetric framework, delimiting hexagonal tunnels along the [100] direction, in which the $\mathrm{K}^{+}$cations are located (Fig. 5). The potassium cations are distributed over three independent crystallographic positions with partial occupancies

\section{Database survey}

The investigated compound is a new member of the $\beta$-xenophyllite family that includes $\mathrm{Na}_{4} \mathrm{Ni}_{7}\left(\mathrm{PO}_{4}\right)_{6}$ (Moring \& Kostiner, 1986), $\mathrm{Na}_{4} \mathrm{Co}_{7}\left(\mathrm{PO}_{4}\right)_{6}$ (Kobashi et al., 1998),

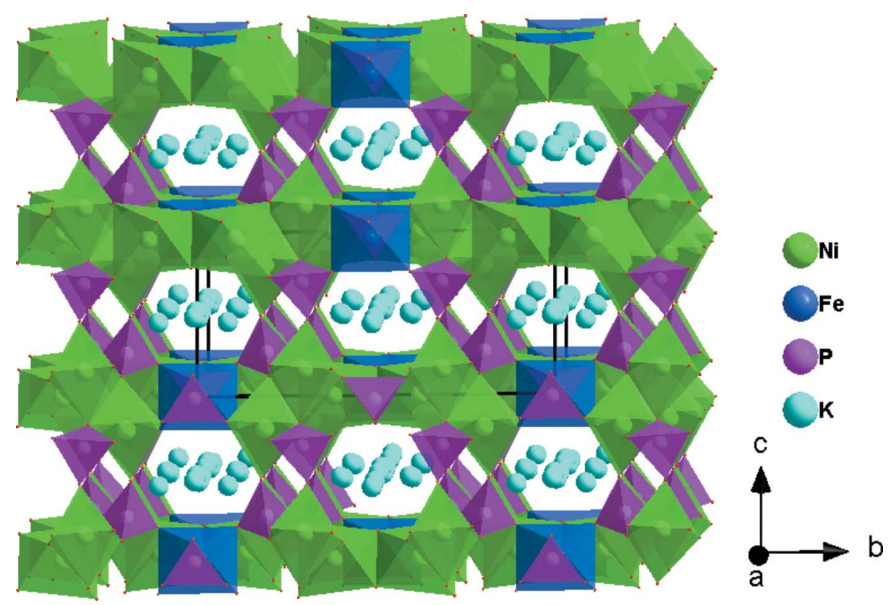

Figure 5

Polyhedral representation of the crystal structure of $\mathrm{K}_{3} \mathrm{Ni}_{6} \mathrm{Fe}\left(\mathrm{PO}_{4}\right)_{6}$ showing large tunnels running along the [100] direction that contain the $\mathrm{K}^{+}$cations. 
Table 1

Experimental details.

\begin{tabular}{|c|c|}
\hline \multicolumn{2}{|l|}{ Crystal data } \\
\hline Chemical formula & $\mathrm{K}_{3} \mathrm{Ni}_{6} \mathrm{Fe}\left(\mathrm{PO}_{4}\right)_{6}$ \\
\hline$M_{\mathrm{r}}$ & 1095.23 \\
\hline Crystal system, space group & Monoclinic, $C 2 / m$ \\
\hline Temperature $(\mathrm{K})$ & 296 \\
\hline$a, b, c(\AA)$ & $10.6853(4), 14.1009(5), 6.5481$ (2) \\
\hline$\beta\left(^{\circ}\right)$ & $103.842(1)$ \\
\hline$V\left(\AA^{3}\right)$ & $957.97(6)$ \\
\hline$Z$ & 2 \\
\hline Radiation type & Мо $K \alpha$ \\
\hline$\mu\left(\mathrm{mm}^{-1}\right)$ & 7.79 \\
\hline Crystal size (mm) & $0.36 \times 0.27 \times 0.20$ \\
\hline \multicolumn{2}{|l|}{ Data collection } \\
\hline Diffractometer & $\begin{array}{l}\text { Bruker D8 VENTURE Super } \\
\text { DUO }\end{array}$ \\
\hline Absorption correction & $\begin{array}{l}\text { Multi-scan (SADABS; Krause et } \\
\quad \text { al., 2015) }\end{array}$ \\
\hline$T_{\min }, T_{\max }$ & $0.638,0.746$ \\
\hline $\begin{array}{l}\text { No. of measured, independent and } \\
\text { observed }[I>2 \sigma(I)] \text { reflections }\end{array}$ & $13870,1981,1891$ \\
\hline$R_{\text {int }}$ & 0.021 \\
\hline$(\sin \theta / \lambda)_{\max }\left(\AA^{-1}\right)$ & 0.781 \\
\hline \multicolumn{2}{|l|}{ Refinement } \\
\hline$R\left[F^{2}>2 \sigma\left(F^{2}\right)\right], w R\left(F^{2}\right), S$ & $0.024,0.062,1.07$ \\
\hline No. of reflections & 1981 \\
\hline No. of parameters & 112 \\
\hline$\Delta \rho_{\max }, \Delta \rho_{\min }\left(\mathrm{e} \AA^{-3}\right)$ & $2.34,-1.16$ \\
\hline
\end{tabular}

Computer programs: APEX3 and SAINT-Plus (Bruker, 2016), SHELXT2014 (Sheldrick, 2015a), SHELXL2016 (Sheldrick, 2015b), ORTEP-3 for Windows (Farrugia, 2012), DIAMOND (Brandenburg, 2006) and publCIF (Westrip, 2010).

$\mathrm{K}_{4} \mathrm{Ni}_{7}\left(\mathrm{AsO}_{4}\right)_{6}$ (Ben Smail et al., 1999), $\mathrm{Na}_{4} \mathrm{Co}_{5.63} \mathrm{Al}_{0.91}\left(\mathrm{AsO}_{4}\right)_{6}$ (Marzouki et al., 2010), $\quad \mathrm{Na}_{4} \mathrm{Li}_{0.62} \mathrm{Co}_{5.67} \mathrm{Al}_{0.71}\left(\mathrm{AsO}_{4}\right)_{6}$ (Marzouki et al., 2013), $\mathrm{Ag}_{4} \mathrm{Co}_{7}\left(\mathrm{AsO}_{4}\right)_{6}$ (Marzouki et al., 2014) and $\mathrm{Na}_{4} \mathrm{Co}_{7}\left(\mathrm{AsO}_{4}\right)_{6}$ (Ben Smida et al., 2016). The phosphates of these compounds crystallize in the non-centrosymmetric $\mathrm{Cm}$ space group while the arsenates adopt the $C 2 / m$ space group.

\section{Synthesis and crystallization}

Single crystals of $\mathrm{K}_{3} \mathrm{Ni}_{6} \mathrm{Fe}\left(\mathrm{PO}_{4}\right)_{6}$ were prepared by solid-state reaction in air. A mixture of $\mathrm{K}_{2} \mathrm{CO}_{3}, \mathrm{Ni}\left(\mathrm{NO}_{3}\right)_{2} \cdot 6 \mathrm{H}_{2} \mathrm{O}$, $\mathrm{Fe}\left(\mathrm{NO}_{3}\right)_{3} \cdot 9 \mathrm{H}_{2} \mathrm{O}$ and $\mathrm{H}_{3} \mathrm{PO}_{4}(85$ wt. \%) reagents with a $\mathrm{K}: \mathrm{Ni}: F e: P$ molar ratio of 2:2:1:3 was dissolved in $50 \mathrm{~mL}$ of distilled water. The resulting solution was stirred without heating for $24 \mathrm{~h}$ and was subsequently evaporated to dryness at $343 \mathrm{~K}$. The obtained dry residue was progressively heated in a platinum crucible up to $673 \mathrm{~K}$ in order to eliminate volatile products. In a second step, the powder was homogenized in an agate mortar and then progressively heated to $1303 \mathrm{~K}$. Kept at this temperature for $2 \mathrm{~h}$, the reaction mixture then underwent slow cooling at a rate of $5 \mathrm{Kh}^{-1}$ to $1103 \mathrm{~K}$ and then to room temperature with the furnace inertia. After washing with distilled water, the obtained crystals were brown with block- type shape. A qualitative EDX analysis (energy dispersive $\mathrm{X}$-ray spectroscopy) detected the presence of the expected chemical elements corresponding to $\mathrm{K}, \mathrm{Ni}, \mathrm{Fe}, \mathrm{P}$ and $\mathrm{O}$ atoms (see Fig. 6).

\section{Refinement}

Crystal data, data collection and structure refinement details of $\mathrm{K}_{3} \mathrm{Ni}_{6} \mathrm{Fe}\left(\mathrm{PO}_{4}\right)_{6}$ are summarized in Table 1 . The highest peak and the deepest hole in the final Fourier map are at 0.71 and $0.59 \AA$, respectively, from atom K2.

\section{Acknowledgements}

The authors thank the Faculty of Science of the Mohammed V University in Rabat, Morocco, for the X-ray measurements.

\section{References}

Ben Smail, R., Driss, A. \& Jouini, T. (1999). Acta Cryst. C55, 284-286. Ben Smida, Y., Marzouki, R., Georges, S., Kutteh, R., Avdeev, M., Guesmi, A. \& Zid, M. F. (2016). J. Solid State Chem. 239, 8-16.

Brandenburg, K. (2006). DIAMOND. Crystal Impact GbR, Bonn, Germany.

Bruker (2016). APEX3, SAINT-Plus and SADABS. Bruker AXS Inc., Madison, Wisconsin, USA.

Essehli, R., Belharouak, I., Ben Yahia, H., Chamoun, R., Orayech, B., El Bali, B., Bouziane, K., Zhou, X. L. \& Zhou, Z. (2015). Dalton Trans. 44, 4526-4532.

Farrugia, L. J. (2012). J. Appl. Cryst. 45, 849-854.

Hatert, F., Long, G. J., Hautot, D., Fransolet, A. M., Delwiche, J., Hubin-Franskin, M. J. \& Grandjean, F. (2004). Phys. Chem. Miner. 31, 487-506.

Kobashi, D., Kohara, S., Yamakawa, J. \& Kawahara, A. (1998). Acta Cryst. C54, 7-9.

Krause, L., Herbst-Irmer, R., Sheldrick, G. M. \& Stalke, D. (2015). J. Appl. Cryst. 48, 3-10.

La Parola, V., Liveri, V. T., Todaro, L., Lombardo, D., Bauer, E. M., Dell'Era, A., Longo, A., Caschera, D., de Caro, T., Toro, R. G. \& Calandra, P. (2018). Mater. Lett. 220, 58-61.

Lazoryak, B. I., Morozov, V. A., Belik, A. A., Stefanovich, S. Y., Grebenev, V. V., Leonidov, I. A., Mitberg, E. B., Davydov, S. A., Lebedev, O. I. \& Van Tendeloo, G. (2004). Solid State Sci. 6, 185195.

Marzouki, R., Frigui, W., Guesmi, A., Zid, M. F. \& Driss, A. (2013). Acta Cryst. E69, i65-i66.

Marzouki, R., Guesmi, A. \& Driss, A. (2010). Acta Cryst. C66, i95i98.

Marzouki, R., Guesmi, A., Georges, S., Zid, M. F. \& Driss, A. (2014). J. Alloys Compd. 586, 74-79.

Moffat, J. B. (1978). Catal. Rev. 18, 199-258.

Moring, J. \& Kostiner, E. (1986). J. Solid State Chem. 62, 105-111.

Orikasa, Y., Gogyo, Y., Yamashige, H., Katayama, M., Chen, K., Mori, T., Yamamoto, K., Masese, T., Inada, Y., Ohta, T., Siroma, Z., Kato, S., Kinoshita, H., Arai, H., Ogumi, Z. \& Uchimoto, Y. (2016). Sci. Rep. 6, article No. 26382.

Saw, L. H., Somasundaram, K., Ye, Y. \& Tay, A. A. O. (2014). J. Power Sources, 249, 231-238.

Sheldrick, G. M. (2015a). Acta Cryst. A71, 3-8.

Sheldrick, G. M. (2015b). Acta Cryst. C71, 3-8.

Westrip, S. P. (2010). J. Appl. Cryst. 43, 920-925. 


\section{supporting information}

Acta Cryst. (2019). E75, 402-404 [https://doi.org/10.1107/S2056989019002706]

\section{Crystal structure of a new tripotassium hexanickel iron hexaphosphate}

\section{Said Ouaatta, Abderrazzak Assani, Mohamed Saadi and Lahcen El Ammari}

Computing details

Data collection: APEX3 (Bruker, 2016); cell refinement: SAINT-Plus (Bruker, 2016); data reduction: SAINT-Plus (Bruker, 2016); program(s) used to solve structure: SHELXT2014 (Sheldrick, 2015a); program(s) used to refine structure:

SHELXL2016 (Sheldrick, 2015b); molecular graphics: ORTEP-3 for Windows (Farrugia, 2012) and DIAMOND

(Brandenburg, 2006); software used to prepare material for publication: publCIF (Westrip, 2010).

Tripotassium hexanickel iron hexaphosphate

Crystal data

$\mathrm{K}_{3} \mathrm{Ni}_{6} \mathrm{Fe}\left(\mathrm{PO}_{4}\right)_{6}$

$M_{r}=1095.23$

Monoclinic, $C 2 / m$

$a=10.6853(4) \AA$

$b=14.1009(5) \AA$

$c=6.5481(2) \AA$

$\beta=103.842(1)^{\circ}$

$V=957.97(6) \AA^{3}$

$Z=2$

Data collection

Bruker D8 VENTURE Super DUO diffractometer

Radiation source: INCOATEC I $\mu \mathrm{S}$ micro-focus source

HELIOS mirror optics monochromator

Detector resolution: 10.4167 pixels $\mathrm{mm}^{-1}$

$\varphi$ and $\omega$ scans

Absorption correction: multi-scan

(SADABS; Krause et al., 2015)

\section{Refinement}

Refinement on $F^{2}$

Least-squares matrix: full

$R\left[F^{2}>2 \sigma\left(F^{2}\right)\right]=0.024$

$w R\left(F^{2}\right)=0.062$

$S=1.07$

1981 reflections

112 parameters
$F(000)=1066$

$D_{\mathrm{x}}=3.797 \mathrm{Mg} \mathrm{m}^{-3}$

Mo $K \alpha$ radiation, $\lambda=0.71073 \AA$

Cell parameters from 1981 reflections

$\theta=2.4-33.7^{\circ}$

$\mu=7.79 \mathrm{~mm}^{-1}$

$T=296 \mathrm{~K}$

Block, brown

$0.36 \times 0.27 \times 0.20 \mathrm{~mm}$

$T_{\min }=0.638, T_{\max }=0.746$

13870 measured reflections

1981 independent reflections

1891 reflections with $I>2 \sigma(I)$

$R_{\text {int }}=0.021$

$\theta_{\text {max }}=33.7^{\circ}, \theta_{\min }=2.4^{\circ}$

$h=-14 \rightarrow 16$

$k=-22 \rightarrow 22$

$l=-10 \rightarrow 10$

0 restraints

$w=1 /\left[\sigma^{2}\left(F_{\mathrm{o}}^{2}\right)+(0.0262 P)^{2}+6.1957 P\right]$

where $P=\left(F_{\mathrm{o}}^{2}+2 F_{\mathrm{c}}^{2}\right) / 3$

$(\Delta / \sigma)_{\max }=0.001$

$\Delta \rho_{\max }=2.34$ e $\AA^{-3}$

$\Delta \rho_{\min }=-1.16 \mathrm{e} \AA^{-3}$ 


\section{Special details}

Geometry. All esds (except the esd in the dihedral angle between two 1.s. planes) are estimated using the full covariance matrix. The cell esds are taken into account individually in the estimation of esds in distances, angles and torsion angles; correlations between esds in cell parameters are only used when they are defined by crystal symmetry. An approximate (isotropic) treatment of cell esds is used for estimating esds involving l.s. planes.

Fractional atomic coordinates and isotropic or equivalent isotropic displacement parameters $\left(\hat{A}^{2}\right)$

\begin{tabular}{llllll}
\hline & $x$ & $y$ & $z$ & $U_{\text {iso }} * / U_{\text {eq }}$ & Occ. $(<1)$ \\
\hline Ni1 & $0.33034(2)$ & $0.69342(2)$ & $0.18429(4)$ & $0.00599(6)$ & \\
Ni2 & 0.500000 & $0.87799(2)$ & 1.000000 & $0.00683(7)$ & \\
Fe1 & 0.500000 & 0.500000 & 0.000000 & $0.00327(9)$ & \\
K1 & $0.40983(18)$ & 0.500000 & $0.4730(2)$ & $0.0200(3)$ & 0.472 \\
K2 & $0.38224(13)$ & $0.90311(11)$ & $0.48618(19)$ & $0.0242(3)$ & 0.417 \\
K3 & $0.1849(7)$ & 0.500000 & $0.4893(8)$ & $0.0560(19)$ & 0.192 \\
P1 & $0.41063(4)$ & $0.69522(3)$ & $0.72442(7)$ & $0.00485(8)$ & \\
P2 & $0.19569(6)$ & 0.500000 & $-0.01061(10)$ & $0.00557(11)$ & \\
O1 & $0.31025(13)$ & $0.70594(10)$ & $0.8587(2)$ & $0.0072(2)$ & \\
O2 & $0.34525(14)$ & $0.68172(11)$ & $0.4959(2)$ & $0.0102(2)$ & \\
O3 & $0.50488(13)$ & $0.60977(10)$ & $0.7970(2)$ & $0.0069(2)$ & \\
O4 & $0.50525(13)$ & $0.78185(10)$ & $0.7692(2)$ & $0.0082(2)$ & \\
O5 & $0.19430(13)$ & $0.58983(10)$ & $0.1265(2)$ & $0.0085(2)$ & \\
O6 & $0.06136(18)$ & 0.500000 & $-0.1681(3)$ & $0.0087(3)$ & \\
O7 & $0.30728(19)$ & 0.500000 & $-0.1148(3)$ & $0.0103(3)$ & \\
& & & & & \\
\hline
\end{tabular}

Atomic displacement parameters $\left(\AA^{2}\right)$

\begin{tabular}{lllllll}
\hline & $U^{11}$ & $U^{22}$ & $U^{33}$ & $U^{12}$ & $U^{13}$ & $U^{23}$ \\
\hline Ni1 & $0.00441(10)$ & $0.00697(10)$ & $0.00638(10)$ & $0.00038(7)$ & $0.00088(7)$ & $-0.00015(7)$ \\
Ni2 & $0.00519(13)$ & $0.00602(14)$ & $0.00944(14)$ & 0.000 & $0.00207(10)$ & 0.000 \\
Fe1 & $0.00218(18)$ & $0.00293(18)$ & $0.00478(19)$ & 0.000 & $0.00099(15)$ & 0.000 \\
K1 & $0.0413(9)$ & $0.0080(5)$ & $0.0103(5)$ & 0.000 & $0.0055(5)$ & 0.000 \\
K2 & $0.0259(6)$ & $0.0332(7)$ & $0.0122(4)$ & $-0.0107(5)$ & $0.0018(4)$ & $0.0082(4)$ \\
K3 & $0.056(4)$ & $0.100(6)$ & $0.0100(17)$ & 0.000 & $0.005(2)$ & 0.000 \\
P1 & $0.00395(17)$ & $0.00570(18)$ & $0.00483(18)$ & $0.00067(13)$ & $0.00093(14)$ & $-0.00035(13)$ \\
P2 & $0.0033(2)$ & $0.0049(2)$ & $0.0082(3)$ & 0.000 & $0.00071(19)$ & 0.000 \\
O1 & $0.0055(5)$ & $0.0095(5)$ & $0.0072(5)$ & $0.0022(4)$ & $0.0028(4)$ & $0.0009(4)$ \\
O2 & $0.0091(6)$ & $0.0162(6)$ & $0.0048(5)$ & $0.0011(5)$ & $0.0003(4)$ & $0.0002(4)$ \\
O3 & $0.0049(5)$ & $0.0070(5)$ & $0.0089(5)$ & $0.0014(4)$ & $0.0018(4)$ & $0.0012(4)$ \\
O4 & $0.0069(5)$ & $0.0072(5)$ & $0.0110(6)$ & $-0.0012(4)$ & $0.0031(4)$ & $-0.0022(4)$ \\
O5 & $0.0071(5)$ & $0.0067(5)$ & $0.0120(6)$ & $-0.0012(4)$ & $0.0027(4)$ & $-0.0031(4)$ \\
O6 & $0.0045(7)$ & $0.0124(8)$ & $0.0081(8)$ & 0.000 & $-0.0005(6)$ & 0.000 \\
O7 & $0.0056(7)$ & $0.0088(8)$ & $0.0181(9)$ & 0.000 & $0.0060(7)$ & 0.000 \\
& & & & & &
\end{tabular}

Geometric parameters $\left(\AA,{ }^{\circ}\right)$

\begin{tabular}{llll}
\hline $\mathrm{Ni} 1-\mathrm{O} 2$ & $2.0153(14)$ & $\mathrm{K} 1-\mathrm{O} 7^{\mathrm{xi}}$ & $3.146(3)$ \\
$\mathrm{Ni} 1-\mathrm{O} 5$ & $2.0314(14)$ & $\mathrm{K} 2-\mathrm{O} 4$ & $2.631(2)$
\end{tabular}




$$
\begin{aligned}
& \mathrm{Ni} 1-\mathrm{O} 1^{\mathrm{i}} \\
& \mathrm{Ni} 1-\mathrm{O}^{\mathrm{ii}} \\
& \mathrm{Ni} 1-\mathrm{O} 1^{\mathrm{iii}} \\
& \mathrm{Ni} 1-\mathrm{O} 4^{\mathrm{ii}} \\
& \mathrm{Ni} 2-\mathrm{O} 4 \\
& \mathrm{Ni2}-\mathrm{O} 4^{\text {iv }} \\
& \mathrm{Ni} 2-\mathrm{O}^{\mathrm{v}} \\
& \mathrm{Ni} 2-\mathrm{O}^{\mathrm{i}} \\
& \mathrm{Ni} 2-\mathrm{O}^{\mathrm{i}} \\
& \mathrm{Ni} 2-\mathrm{O}^{\mathrm{vi}} \\
& \mathrm{Fe} 1-\mathrm{O} 7 \\
& \mathrm{Fe} 1-\mathrm{O}^{\text {vii }} \\
& \mathrm{Fe} 1-\mathrm{O}^{\text {viii }} \\
& \mathrm{Fe} 1-\mathrm{O}^{3 i} \\
& \mathrm{Fe} 1-\mathrm{O}^{\text {ix }} \\
& \mathrm{Fe} 1-\mathrm{O}^{\text {iii }} \\
& \mathrm{K} 1-\mathrm{O} 3 \\
& \mathrm{~K} 1-\mathrm{O}^{\mathrm{x}} \\
& \mathrm{K} 1-\mathrm{O} 2 \\
& \mathrm{~K} 1-\mathrm{O}^{\mathrm{x}} \\
& \mathrm{K} 1-\mathrm{O} 3^{\mathrm{ix}} \\
& \mathrm{K} 1-\mathrm{O}^{\mathrm{ii}} \\
& \mathrm{K} 1-\mathrm{O} 5 \\
& \mathrm{~K} 1-\mathrm{O}^{\mathrm{x}} \\
& \mathrm{O} 2-\mathrm{Ni} 1-\mathrm{O} 5 \\
& \mathrm{O} 2-\mathrm{Ni1}-\mathrm{O}^{\mathrm{i}} \\
& \mathrm{O} 5-\mathrm{Ni} 1-\mathrm{O}^{\mathrm{i}} \\
& \mathrm{O} 2-\mathrm{Ni} 1-\mathrm{O}^{3 i} \\
& \mathrm{O} 5-\mathrm{Ni} 1-\mathrm{O}^{\mathrm{ii}} \\
& \mathrm{O} 1^{\mathrm{i}}-\mathrm{Ni} 1-\mathrm{O} 3^{\mathrm{ii}} \\
& \mathrm{O} 2-\mathrm{Ni1}-\mathrm{O} 1^{\mathrm{iii}} \\
& \mathrm{O} 5-\mathrm{Ni} 1-\mathrm{O}{ }^{\mathrm{iii}}
\end{aligned}
$$

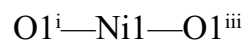

$$
\begin{aligned}
& \mathrm{O}^{3 i}-\mathrm{Ni} 1-\mathrm{O}^{\mathrm{iii}} \\
& \mathrm{O} 2-\mathrm{Ni} 1-\mathrm{O}_{4}^{\mathrm{ii}} \\
& \mathrm{O} 5-\mathrm{Ni} 1-\mathrm{O}^{\mathrm{ii}} \\
& \mathrm{O} 1^{\mathrm{i}}-\mathrm{Ni} 1-\mathrm{O} 4^{\mathrm{ii}}
\end{aligned}
$$

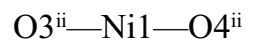

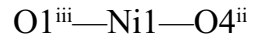

$$
\begin{aligned}
& \mathrm{O} 4-\mathrm{Ni} 2-\mathrm{O}^{\mathrm{iv}} \\
& \mathrm{O} 4-\mathrm{Ni} 2-\mathrm{O}^{\mathrm{v}} \\
& \mathrm{O} 4^{\mathrm{iv}}-\mathrm{Ni} 2-\mathrm{O}^{\mathrm{v}} \\
& \mathrm{O} 4-\mathrm{Ni} 2-\mathrm{O}^{\mathrm{i}} \\
& \mathrm{O} 4^{\mathrm{iv}}-\mathrm{Ni2}-\mathrm{O} 5^{\mathrm{i}} \\
& \mathrm{O} 5^{\mathrm{v}}-\mathrm{Ni} 2-\mathrm{O} 5^{\mathrm{i}} \\
& \mathrm{O} 4-\mathrm{Ni} 2-\mathrm{O}^{\mathrm{i}} \\
& \mathrm{O} 4^{\mathrm{iv}}-\mathrm{Ni} 2-\mathrm{O}^{\mathrm{i}}
\end{aligned}
$$

$2.0366(13)$

$2.0984(14)$

$2.0988(14)$

2.1161 (14)

$2.0411(14)$

$2.0411(14)$

$2.0928(14)$

\begin{tabular}{|c|c|}
\hline $\mathrm{O} 3^{\mathrm{ix}}-\mathrm{K} 1-\mathrm{O} 5$ & $93.66(6)$ \\
\hline $\mathrm{O} 33^{\mathrm{ii}}-\mathrm{K} 1-\mathrm{O} 5$ & $65.70(5)$ \\
\hline $\mathrm{O} 3-\mathrm{K} 1-\mathrm{O}^{\mathrm{x}}$ & $155.60(8)$ \\
\hline $\mathrm{O} 3^{\mathrm{x}}-\mathrm{K} 1-\mathrm{O}^{\mathrm{x}}$ & $115.32(5)$ \\
\hline $\mathrm{O} 2-\mathrm{K} 1-\mathrm{O} 5^{\mathrm{x}}$ & $106.12(6)$ \\
\hline $\mathrm{O} 2^{\mathrm{x}}-\mathrm{K} 1-\mathrm{O} 5^{\mathrm{x}}$ & $59.36(5)$ \\
\hline $\mathrm{O} 3^{\mathrm{ix}}-\mathrm{K} 1-\mathrm{O} 5^{\mathrm{x}}$ & $65.70(5)$ \\
\hline $\mathrm{O} 3^{\mathrm{ii}}-\mathrm{K} 1-\mathrm{O}^{\mathrm{x}}$ & $93.66(6)$ \\
\hline $\mathrm{O} 5-\mathrm{K} 1-\mathrm{O} 5^{\mathrm{x}}$ & $48.38(6)$ \\
\hline $\mathrm{O} 3-\mathrm{K} 1-\mathrm{O} 7^{\mathrm{xi}}$ & $56.89(5)$ \\
\hline $\mathrm{O} 3^{\mathrm{x}}-\mathrm{K} 1-\mathrm{O} 7^{\mathrm{xi}}$ & $56.89(5)$ \\
\hline $\mathrm{O} 2-\mathrm{K} 1-\mathrm{O}^{\mathrm{xi}}$ & $78.69(5)$ \\
\hline $\mathrm{O} 2^{\mathrm{x}}-\mathrm{K} 1-\mathrm{O} 7^{\mathrm{xi}}$ & $78.69(5)$ \\
\hline $\mathrm{O} 3^{\mathrm{ix}}-\mathrm{K} 1-\mathrm{O} 7^{\mathrm{xi}}$ & $144.33(3)$ \\
\hline $\mathrm{O} 3^{\mathrm{ii}-}-\mathrm{K} 1-\mathrm{O} 7^{\mathrm{xi}}$ & $144.33(3)$ \\
\hline $\mathrm{O} 5-\mathrm{K} 1-\mathrm{O} 7^{\mathrm{xi}}$ & $106.17(7)$ \\
\hline $\mathrm{O}^{\mathrm{x}}-\mathrm{K} 1-\mathrm{O} 7^{\mathrm{xi}}$ & $106.17(7)$ \\
\hline $\mathrm{O} 4-\mathrm{K} 2-\mathrm{O}^{\mathrm{xii}}$ & $135.41(8)$ \\
\hline $\mathrm{O} 4-\mathrm{K} 2-\mathrm{O} 2^{\mathrm{i}}$ & $89.14(6)$ \\
\hline $\mathrm{O} 6^{\mathrm{xii}}-\mathrm{K} 2-\mathrm{O} 2^{\mathrm{i}}$ & $128.60(7)$ \\
\hline $\mathrm{O} 4-\mathrm{K} 2-\mathrm{O} 5^{\mathrm{i}}$ & $66.22(5)$ \\
\hline $\mathrm{O} 6^{\mathrm{xii}}-\mathrm{K} 2-\mathrm{O} 5^{\mathrm{i}}$ & $147.28(7)$ \\
\hline $\mathrm{O} 2^{\mathrm{i}}-\mathrm{K} 2-\mathrm{O} 5^{\mathrm{i}}$ & $61.95(5)$ \\
\hline
\end{tabular}

2.0928 (14)

$2.2241(13)$

$2.2241(13)$

$2.016(2)$

$2.016(2)$

$2.0490(13)$

$2.0490(13)$

$2.0490(13)$

$2.0490(13)$

$2.6263(18)$

$2.6264(18)$

$2.6673(16)$

$2.6673(16)$

$2.6691(19)$

$2.6691(19)$

$3.091(2)$

3.091 (2)

$90.55(6)$

$94.23(6)$

$90.23(6)$

$91.89(6)$

$99.19(5)$

$168.72(5)$

$178.70(6)$

$88.65(6)$

$84.74(6)$

$89.25(5)$

$92.33(6)$

$169.40(5)$

$99.72(5)$

$70.53(5)$

88.64 (5)

$96.75(8)$

$103.69(6)$

92.95 (5)

$92.95(5)$

103.69 (6)

$154.96(8)$

160.71 (6)

$94.81(6)$

$$
\begin{aligned}
& \mathrm{K} 2-\mathrm{O}^{\mathrm{xii}} \\
& \mathrm{K} 2-2^{\mathrm{i}} \\
& \mathrm{K} 2-\mathrm{O}^{\mathrm{i}} \\
& \mathrm{K} 2-\mathrm{O}^{4 i} \\
& \mathrm{~K} 2-6^{\mathrm{ii}} \\
& \mathrm{K} 2-\mathrm{O}^{\mathrm{i}} \\
& \mathrm{K} 2-\mathrm{O} 7^{\mathrm{xii}} \\
& \mathrm{K} 2-\mathrm{O} 2 \\
& \mathrm{~K} 3-\mathrm{O} 7^{\mathrm{xi}} \\
& \mathrm{K} 3-\mathrm{O} 5 \\
& \mathrm{~K} 3-\mathrm{O} 5^{\mathrm{x}} \\
& \mathrm{K} 3-\mathrm{O} 6^{\mathrm{xi}} \\
& \mathrm{K} 3-\mathrm{O} 6^{\mathrm{xii}} \\
& \mathrm{K} 3-\mathrm{O} 2 \\
& \mathrm{~K} 3-\mathrm{O} 2^{\mathrm{x}} \\
& \mathrm{P} 1-\mathrm{O} 2 \\
& \mathrm{P} 1-\mathrm{O} 1 \\
& \mathrm{P} 1-\mathrm{O} 4 \\
& \mathrm{P} 1-\mathrm{O} 3 \\
& \mathrm{P} 2-\mathrm{O} 7 \\
& \mathrm{P} 2-\mathrm{O} 6 \\
& \mathrm{P} 2-\mathrm{O} 5^{\mathrm{x}} \\
& \mathrm{P} 2-\mathrm{O} 5
\end{aligned}
$$

2.677 (2)

2.737 (2)

2.8470 (19)

2.851 (2)

2.929 (2)

3.0776 (19)

3.086 (2)

3.149 (2)

2.609 (6)

$2.715(5)$

$2.715(5)$

$2.862(6)$

2.949 (7)

3.077 (4)

3.077 (4)

1.5042 (15)

1.5482 (14)

1.5679 (14)

1.5694 (14)

1.509 (2)

1.554 (2)

1.5546 (14)

1.5546 (14)

$93.66(6)$

$65.70(5)$

$155.60(8)$

$115.32(5)$

$106.12(6)$

$59.36(5)$

$65.70(5)$

$93.66(6)$

$48.38(6)$

$56.89(5)$

$56.89(5)$

8.69 (5)

144.33 (3)

144.33 (3)

06.17 (7)

106.17 (7)

$135.41(8)$

89.14 (6)

$128.60(7)$

$147.28(7)$

$61.95(5)$ 


$$
\begin{aligned}
& \mathrm{O} 5^{\mathrm{v}}-\mathrm{Ni} 2-\mathrm{O} 6^{\mathrm{i}} \\
& \mathrm{O} 5^{\mathrm{i}}-\mathrm{Ni} 2-\mathrm{O}^{\mathrm{i}} \\
& \mathrm{O} 4-\mathrm{Ni} 2-\mathrm{O}^{\mathrm{vi}} \\
& \mathrm{O} 4^{\mathrm{iv}}-\mathrm{Ni} 2-\mathrm{O}^{\mathrm{vi}} \\
& \mathrm{O} 5^{\mathrm{v}}-\mathrm{Ni} 2-\mathrm{O}^{\mathrm{vi}} \\
& \mathrm{O} 5-\mathrm{Ni} 2-\mathrm{O}^{\mathrm{ii}} \\
& \mathrm{O} 6^{\mathrm{i}}-\mathrm{Ni} 2-\mathrm{O}^{\mathrm{vi}} \\
& \mathrm{O} 7-\mathrm{Fe} 1-\mathrm{O} 7^{\mathrm{vii}} \\
& \mathrm{O} 7-\mathrm{Fe} 1-\mathrm{O}^{\text {viii }} \\
& \mathrm{O} 7^{\mathrm{vii}-\mathrm{Fe} 1-\mathrm{O}^{\text {viii }}} \\
& \mathrm{O} 7-\mathrm{Fe} 1-\mathrm{O}^{3 i} \\
& \text { O7 }{ }^{\text {vii }-F e 1-O ~} 3^{\text {ii }} \\
& \mathrm{O}^{\text {viii }-\mathrm{Fe} 1-\mathrm{O}^{3 i}} \\
& \mathrm{O} 7-\mathrm{Fe} 1-\mathrm{O}^{\text {ix }} \\
& \mathrm{O} 7^{\text {vii }-\mathrm{Fe} 1-\mathrm{O} 3^{\text {ix }}}
\end{aligned}
$$

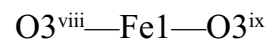

$$
\begin{aligned}
& \mathrm{O}^{3 i}-\mathrm{Fe} 1-\mathrm{O}^{\mathrm{ix}} \\
& \mathrm{O} 7-\mathrm{Fe} 1-\mathrm{O}^{3 i i} \\
& \mathrm{O} 7^{\mathrm{vii}}-\mathrm{Fe} 1-\mathrm{O}^{\text {iii }} \\
& \mathrm{O}^{\text {viii }}-\mathrm{Fe} 1-\mathrm{O}^{\text {iii }} \\
& \mathrm{O}^{3 i}-\mathrm{Fe} 1-\mathrm{O}^{i i i} \\
& \mathrm{O}^{3}{ }^{\text {ix }}-\mathrm{Fe} 1-\mathrm{O} 3^{\text {iii }} \\
& \mathrm{O} 2-\mathrm{P} 1-\mathrm{O} 1 \\
& \mathrm{O} 2-\mathrm{P} 1-\mathrm{O} 4 \\
& \mathrm{O} 1-\mathrm{P} 1-\mathrm{O} 4 \\
& \mathrm{O} 2-\mathrm{P} 1-\mathrm{O} 3 \\
& \mathrm{O} 1-\mathrm{P} 1-\mathrm{O} 3 \\
& \mathrm{O} 4-\mathrm{P} 1-\mathrm{O} 3 \\
& \mathrm{O} 7-\mathrm{P} 2-\mathrm{O} 6 \\
& \mathrm{O} 7-\mathrm{P} 2-\mathrm{O}^{\mathrm{x}} \\
& \mathrm{O} 6-\mathrm{P} 2-\mathrm{O} 5^{\mathrm{x}} \\
& \mathrm{O} 7-\mathrm{P} 2-\mathrm{O} 5 \\
& \text { O6- } 2 \text { 2-O5 } \\
& \mathrm{O} 5 \text { - }-\mathrm{P} 2-\mathrm{O} 5 \\
& \mathrm{O} 3-\mathrm{K} 1-\mathrm{O}^{\mathrm{x}} \\
& \mathrm{O} 3-\mathrm{K} 1-\mathrm{O} 2 \\
& \mathrm{O} 3^{\mathrm{x}}-\mathrm{K} 1-\mathrm{O} 2 \\
& \mathrm{O} 3-\mathrm{K} 1-\mathrm{O} 2^{\mathrm{x}} \\
& \mathrm{O} 3^{\mathrm{x}}-\mathrm{K} 1-\mathrm{O} 2^{\mathrm{x}} \\
& \mathrm{O} 2-\mathrm{K} 1-\mathrm{O} 2^{\mathrm{x}} \\
& \mathrm{O} 3-\mathrm{K} 1-\mathrm{O}^{\text {ix }} \\
& \mathrm{O} 3^{\mathrm{x}}-\mathrm{K} 1-\mathrm{O}^{\mathrm{ix}} \\
& \mathrm{O} 2-\mathrm{K} 1-\mathrm{O} 3^{\text {ix }} \\
& \mathrm{O} 2^{\mathrm{x}}-\mathrm{K} 1-\mathrm{O}^{\mathrm{ix}} \\
& \mathrm{O} 3-\mathrm{K} 1-\mathrm{O} 3^{\mathrm{ii}} \\
& \mathrm{O} 3^{\mathrm{x}}-\mathrm{K} 1-\mathrm{O}^{\mathrm{ii}} \\
& \mathrm{O} 2-\mathrm{K} 1-\mathrm{O} 3^{\mathrm{ii}} \\
& \mathrm{O} 2^{\mathrm{x}}-\mathrm{K} 1-\mathrm{O}^{3 i}
\end{aligned}
$$

$$
\begin{aligned}
& \mathrm{O} 4-\mathrm{K} 2-\mathrm{O} 4^{\mathrm{ii}}
\end{aligned}
$$

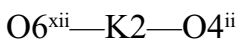

$$
\begin{aligned}
& \mathrm{K} 2^{\mathrm{xi}}-\mathrm{K} 2-\mathrm{O} 4^{\mathrm{ii}} \\
& \mathrm{O} 2^{\mathrm{i}}-\mathrm{K} 2-\mathrm{O} 4^{\mathrm{ii}} \\
& \mathrm{O} 5^{\mathrm{i}}-\mathrm{K} 2-\mathrm{O} 4^{\mathrm{ii}} \\
& \mathrm{O} 4-\mathrm{K} 2-\mathrm{O}^{\mathrm{vi}}
\end{aligned}
$$

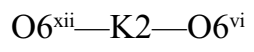

$$
\begin{aligned}
& \mathrm{O} 2^{\mathrm{i}}-\mathrm{K} 2-\mathrm{O}^{\mathrm{vi}} \\
& \mathrm{O} 5-\mathrm{K} 2-\mathrm{O}^{\mathrm{i}} \\
& \mathrm{O} 4^{\mathrm{ii}}-\mathrm{K} 2-\mathrm{O}^{\mathrm{vi}} \\
& \mathrm{O} 4-\mathrm{K} 2-\mathrm{O} 1^{\mathrm{i}} \\
& \mathrm{O}^{\mathrm{xii}}-\mathrm{K} 2-\mathrm{O}^{\mathrm{i}} \\
& \mathrm{O} 2^{\mathrm{i}}-\mathrm{K} 2-\mathrm{O}^{\mathrm{i}} \\
& \mathrm{O} 5-\mathrm{K} 2-\mathrm{O}^{\mathrm{i}}
\end{aligned}
$$

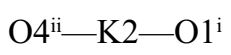

$$
\begin{aligned}
& \mathrm{O}^{\mathrm{vi}}-\mathrm{K} 2-\mathrm{O}^{\mathrm{i}} \\
& \mathrm{O} 4-\mathrm{K} 2-\mathrm{O} 7^{\mathrm{xii}} \\
& \mathrm{O}^{\mathrm{xii}}-\mathrm{K} 2-\mathrm{O} 7^{\mathrm{xii}} \\
& \mathrm{O} 2-\mathrm{i} 2-\mathrm{O} 7^{\mathrm{xii}} \\
& \mathrm{O} 5-\mathrm{K} 2-\mathrm{O} 7^{\mathrm{xii}} \\
& \mathrm{O} 4^{\mathrm{ii}}-\mathrm{K} 2-\mathrm{O} 7^{\mathrm{xii}}
\end{aligned}
$$

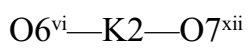

$$
\begin{aligned}
& \mathrm{O} 1^{\mathrm{i}-\mathrm{K} 2} 2-\mathrm{O} 7^{\mathrm{xii}} \\
& \mathrm{O} 4-\mathrm{K} 2-\mathrm{O} 2 \\
& \mathrm{O}^{\mathrm{xii}}-\mathrm{K} 2-\mathrm{O} 2 \\
& \mathrm{O} 2 \mathrm{i}-\mathrm{K} 2-\mathrm{O} 2 \\
& \mathrm{O} 5-\mathrm{K} 2-\mathrm{O} 2 \\
& \mathrm{O} 4 \text { ii- } 2-\mathrm{O} 2 \\
& \mathrm{O}^{\mathrm{vi}}-\mathrm{K} 2-\mathrm{O} 2 \\
& \mathrm{O} 1-\mathrm{i} 2-\mathrm{O} 2 \\
& \mathrm{O} 7{ }^{\mathrm{xi}}-\mathrm{K} 2-\mathrm{O} 2 \\
& \mathrm{O} 7 \mathrm{xi}-\mathrm{K} 3-\mathrm{O} 5 \\
& \mathrm{O} 7^{\mathrm{xi}}-\mathrm{K} 3-\mathrm{O} 5^{\mathrm{x}} \\
& \mathrm{O} 5-\mathrm{K} 3-\mathrm{O}^{\mathrm{x}} \\
& \mathrm{O} 7^{\mathrm{xi}}-\mathrm{K} 3-\mathrm{O} 6^{\mathrm{xi}} \\
& \mathrm{O} 5-\mathrm{K} 3-\mathrm{O}^{\mathrm{xi}} \\
& \mathrm{O} 5^{\mathrm{x}}-\mathrm{K} 3-\mathrm{O} 6^{\mathrm{xi}} \\
& \mathrm{O} 7^{\mathrm{xi}}-\mathrm{K} 3-\mathrm{O} 6^{\mathrm{xiii}} \\
& \mathrm{O} 5-\mathrm{K} 3-\mathrm{O}^{\mathrm{xiii}} \\
& \mathrm{O} 5^{\mathrm{x}}-\mathrm{K} 3-\mathrm{O} 6^{\mathrm{xiii}}
\end{aligned}
$$

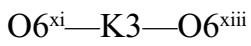

$$
\begin{aligned}
& \mathrm{O} 7 \mathrm{xi}-\mathrm{K} 3-\mathrm{O} 2 \\
& \mathrm{O} 5-\mathrm{K} 3-\mathrm{O} 2 \\
& \mathrm{O} 5{ }^{\mathrm{x}}-\mathrm{K} 3-\mathrm{O} 2 \\
& \mathrm{O} 66^{\mathrm{xi}}-\mathrm{K} 3-\mathrm{O} 2 \\
& \mathrm{O} 6^{\mathrm{xii}-\mathrm{K} 3-\mathrm{O} 2} \\
& \mathrm{O} 7^{\mathrm{xi}}-\mathrm{K} 3-\mathrm{O} 2^{\mathrm{x}} \\
& \mathrm{O} 5-\mathrm{K} 3-\mathrm{O}^{\mathrm{x}}
\end{aligned}
$$

$79.26(7)$

$69.19(5)$

$126.86(4)$

$105.45(6)$

$142.65(7)$

$68.58(5)$

$97.82(7)$

$126.41(7)$

$64.47(5)$

$116.35(6)$

$109.01(6)$

$85.38(5)$

$50.84(4)$

112.77 (6)

$64.63(5)$

$176.79(6)$

$165.00(7)$

$52.30(6)$

$78.76(5)$

$114.35(7)$

$95.32(5)$

$125.92(6)$

$56.34(4)$

$52.08(5)$

$125.05(6)$

$56.55(6)$

$87.27(5)$

$59.33(5)$

$120.57(5)$

56.94 (4)

$113.14(5)$

$139.0(2)$

$139.0(2)$

$55.61(11)$

$55.72(11)$

143.7 (2)

143.7 (2)

149.1 (3)

65.77 (12)

65.77 (12)

93.4 (2)

80.84 (14)

$59.12(9)$

105.29 (19)

110.40 (11)

114.13 (11)

80.84 (14)

105.29 (19) 


$\begin{array}{llll}\mathrm{O}^{2}-\mathrm{K} 1-\mathrm{O} 3^{\mathrm{ii}} & 70.89(7) & \mathrm{O}^{\mathrm{x}}-\mathrm{K} 3-\mathrm{O}^{\mathrm{x}} & 59.12(9) \\ \mathrm{O} 3-\mathrm{K} 1-\mathrm{O} 5 & 115.32(5) & \mathrm{O}^{\mathrm{xi}}-\mathrm{K} 3-\mathrm{O} 2^{\mathrm{x}} & 110.40(11) \\ \mathrm{O} 3^{\mathrm{x}}-\mathrm{K} 1-\mathrm{O} 5 & 155.60(8) & \mathrm{O}^{\mathrm{xiii}}-\mathrm{K} 3-\mathrm{O}^{\mathrm{x}} & 114.13(11) \\ \mathrm{O} 2-\mathrm{K} 1-\mathrm{O} 5 & 59.36(5) & \mathrm{O} 2-\mathrm{K} 3-\mathrm{O}^{\mathrm{x}} & 112.8(2) \\ \mathrm{O} 2^{\mathrm{x}}-\mathrm{K} 1-\mathrm{O} 5 & 106.12(6) & & \end{array}$

Symmetry codes: (i) $-x+1 / 2,-y+3 / 2,-z+1$; (ii) $-x+1, y,-z+1$; (iii) $x, y, z-1$; (iv) $-x+1, y,-z+2$; (v) $x+1 / 2,-y+3 / 2, z+1$; (vi) $x+1 / 2, y+1 / 2, z+1$; (vii) $-x+1$, $-y+1,-z$; (viii) $x,-y+1, z-1$; (ix) $-x+1,-y+1,-z+1$; (x) $x,-y+1, z$; (xi) $x, y, z+1$; (xii) $-x+1 / 2,-y+3 / 2,-z$; (xiii) $-x,-y+1,-z$; (xiv) $x,-y+2, z$. 